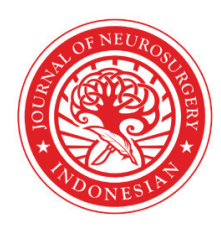

\title{
Management of penetrating brain injury : A case report
}

\author{
Asadullah ${ }^{1 *}$, Endra Wibisono Harmawan'1, Resi Prastikarunia' ${ }^{1}$ Gunna Hutomo Putra', \\ Heru Kustono ${ }^{1}$, Aji Setia Utama ${ }^{1}$, Komang Sena Adistira Artha ${ }^{1}$, \\ Tedy Apriawan², Abdul Hafid Bajamal ${ }^{3}$
}

\begin{abstract}
Introduction: Penetrating brain injury is a medical emergency which, although rare, often results in high mortality and morbidity. Management of penetrating brain injury itself is still a challenge for neurosurgeons around the world. Optimal management of the penetrating brain injury requires a comprehensive understanding of the mechanism and pathophysiology of the occurrence of these injuries. Until now the standard management of cases of penetrating brain trauma is still a debate.

Methods: This article was case series, we reported 3 cases of penetrating trauma at this institution. This article was compiled from various references and experiences of cases that have

been managed at the Dr. Soetomo Academic General Hospital. We got that penetrating brain injury patients who performed debridement craniotomy less than 12 hours post trauma followed by the administration of empirical prophylactic antibiotics with ceftriaxone and metronidazole for 7 days and anti seizure of phenytoin for 7 days.

Results: A satisfactory outcome was obtained.

Conclusion: Management of penetrating brain injury needs early diagnostic methods such as CT scan of the head anf CT angiography for cases of suspected vascular lesions, followed by early operation procedure dan administration of the right antibiotics.
\end{abstract}

Keywords: Penetrating brain injury, debridement craniotomy, empirical prophylactic antibiotics, CT angiography

Cite This Article: Asadullah., Harmawan, E.W., Pratikarunia, R., Putra, G.H., Kustono, H., Utama, A.S., Artha, K.S.A., Apriawan, T., Bajamal, A.H. 2020. Management of penetrating brain injury : A case report. Indonesian Journal of Neurosurgery 3(3): 74-79. D0I: 10.15562/ijn. v3i3.76

'Resident of Neurosurgery Department, dr.Soetomo General Hospital, Surabaya ${ }^{2}$ Staff of Neurosurgery Department, dr.Soetomo General Hospital \& Airlangga University Hospital Surabaya ${ }^{3}$ Professor of Neurosurgery Departement, dr.Soetomo General Hospital, Surabaya

*Corresponding to : Asadullah; Resident of Neurosurgery Department, dr.Soetomo General Hospital, Surabaya; sevelkomodo@gmail.com
Received: 2019-07-19

Accepted: 2020-05-09 Published: 2020-11-19

\section{INTRODUCTION}

Penetrating brain injury (PBI) is any injury where an object pierces the head and meninges that wrap around the brain. ${ }^{1}$ Although the incidence is not as frequent as closed head trauma, penetrating brain trauma has a worse prognosis. ${ }^{2} \mathrm{PBI}$ is the most lifethreatening type of all head trauma. ${ }^{3}$ Only $10 \%$ of patients can survive at the hospital, and half of these patients die in the emergency room while the other half have severe disabilities due to injury. ${ }^{4}$

Based on the speed of penetration, it can be classified into two categories, high-velocity penetration including injuries caused by bullets and low-velocity penetration including injuries caused by a knife or other sharp objects, nail, and scissors. ${ }^{5}$

In society, PBI is usually caused by sharp objects with relatively low velocity in contrast to penetrating brain trauma in a war zone where trauma from firearms is more often encountered at high velocity, and results in more complex injuries and high mortality. ${ }^{2,6}$ Low-velocity brain injury has a better prognosis because the primary injury caused is more localized. ${ }^{2}$

Even though the trauma of the brain penetrates at a low velocity the mortality rate is not high but the complications of post trauma such as infection, cerebrospinal fluid leakage, traumatic aneurysm, intra-ventricular bleeding, sinus thrombosis are serious problems that need to be anticipated. ${ }^{4}$

\section{METHODS}

This research was conducted at the Academic General Hospital Dr. Soetomo. The research design used was a case series with a descriptive approach as the method of translation. The author compares the management done at our institution with the latest literature.

\section{RESULTS}

\section{CASE 1}

A man, 43 years old, came to the emergency room with a complaint that his left eye was punctured by wood. The patient's eye was punctured by wood while sawing 14 hours prior admission to the hospital. Nausea (-), vomiting (-), seizures (-), decreased consciousness (-), limb weakness (-). The patient was consulted from opthalmologisy with a 


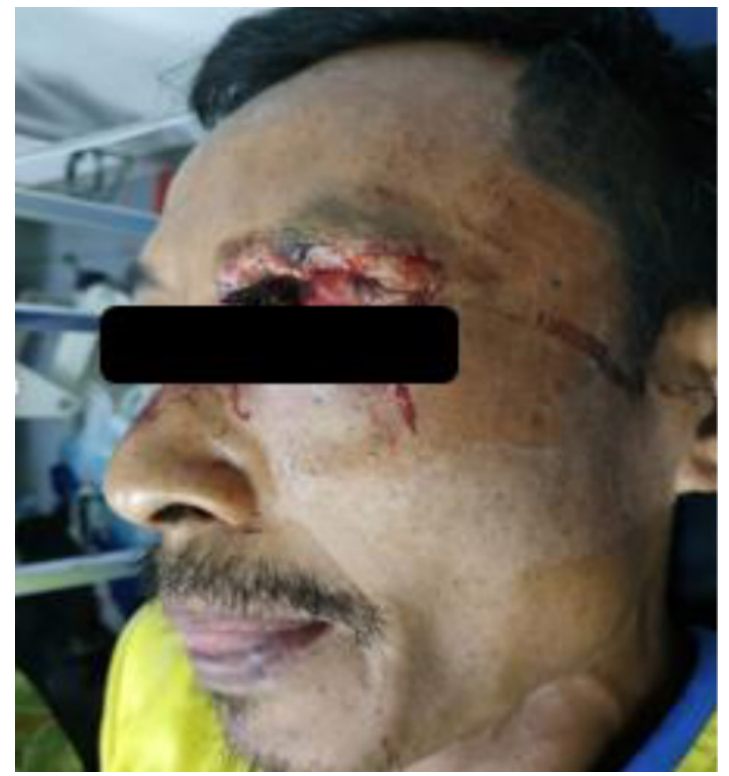

Figure 1. Clinical Photo

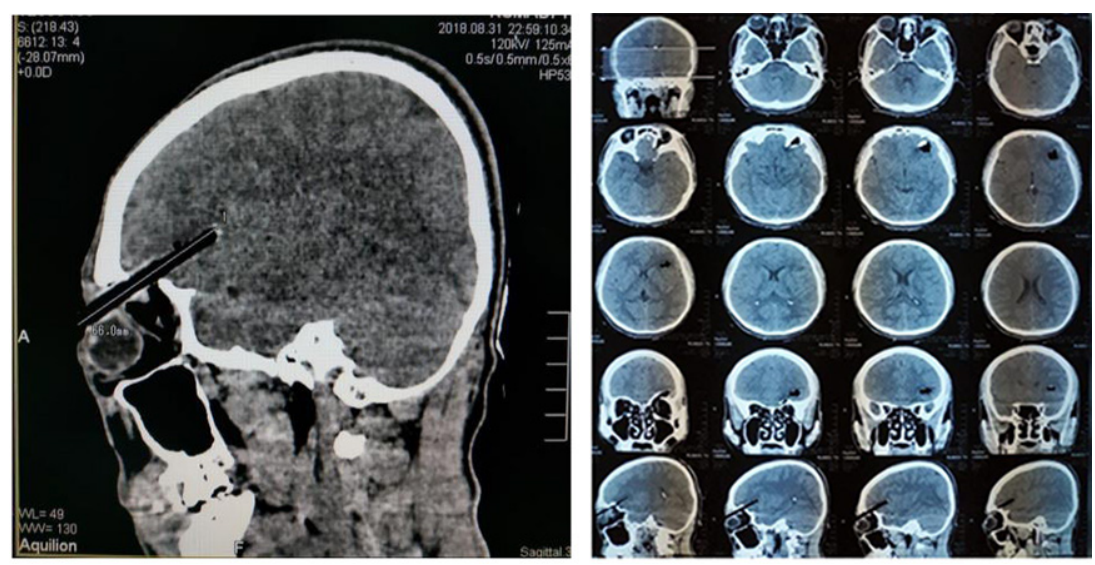

Figure 2. Head CT Scan

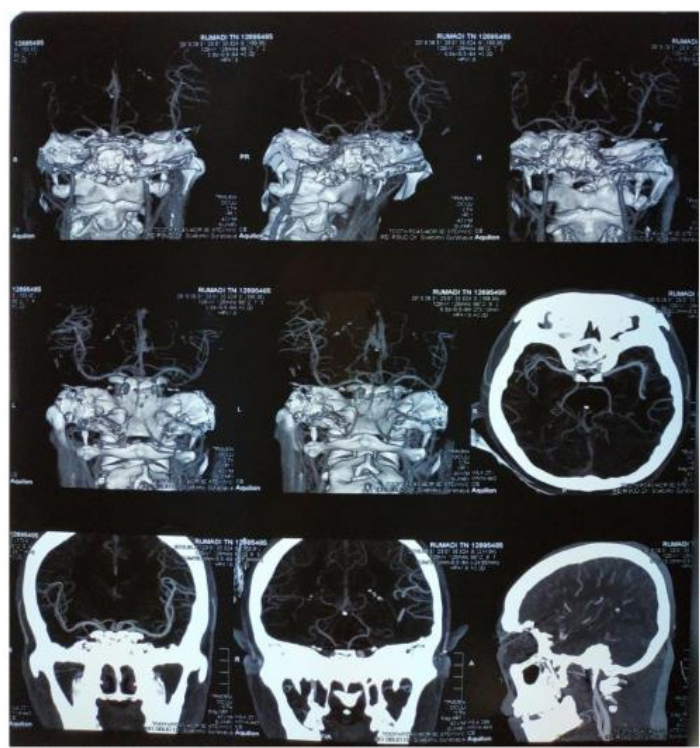

Figure 3. CT Angiography left intra orbital foreign body.

Physical examination, primary survey and hemodynamic status were stable. On local status examination, the entrance wound on the left orbit was obtained and there were still pieces of wood that stick into the patient's eyes. From a neurological examination, GCS 456 was obtained, anisocorous OD $3 \mathrm{~mm}$ OS $6 \mathrm{~mm}$, light reflex right eye $(+)$, left eye decreased, visual acuity right eye $>2 / 60$ left eye light perception (+), meningeal sign (-), other neurological status within normal limitsOn physical examination, primary survey and hemodynamic status were stable. On local status examination, the entrance wound on the left orbit was obtained and there were still pieces of wood that stick into the patient's eyes. From a neurological examination, GCS 456 was obtained, anisocorous OD $3 \mathrm{~mm}$ OS $6 \mathrm{~mm}$, light reflex right eye $(+)$, left eye decreased, visual acuity right eye $>2 / 60$ left eye light perception (+), meningeal sign $(-)$, other neurological status within normal limit.

A head computed tomography (CT) scan without contrast and CT angiography were performed. CT scan showed a $66 \mathrm{~mm}$ piece of wood penetrating through the left eye through the orbital cavity and into the anterior fossa of the intracranial cavity extending to the brain parenchyma. From the axial brain window, there was no visible contusion or cerebral edema, there was a pneumocephalus in the frontal lobe right at the end of the wood. There were no visible fractures and other intracranial objects. From CT angiography, a piece of wood was not exposed to large blood vessels and there was also no traumatic aneurysm.

Patients diagnosed with penetrating cardiac ocular trauma + penetrating trauma of the left frontotemporal brain. Foreign body extraction, craniotomy and debridement were performed. Opthalmologist did a joint surgery performing ocular exploration and palpebral reconstruction.

The patient was positioned supine, disinfected with povidone iodine and the surgical field was narrowed with sterile doek. Design of coronal incisions was made layer by layer followed by skin flaps to supraorbital exposure by preserfation of the supraorbital balance. 6-hole burrhole, craniotomy and hitstich were performed. After the dura exploration was carried out, the foreign body was found to be $7 \mathrm{x} 1.5 \mathrm{~cm}$, wood chips and dura tears were $1.5 \mathrm{~cm}$. The foreign body extraction, debridement and dura sewing were carried out. Bones were returned with miniplate and screw.

Microbiology culture samples were taken when debridement was carried out. Prophylactic empiric antibiotics were given to prevent infection with ceftriaxone inravena $1 \mathrm{~g} / 12$ hours and 


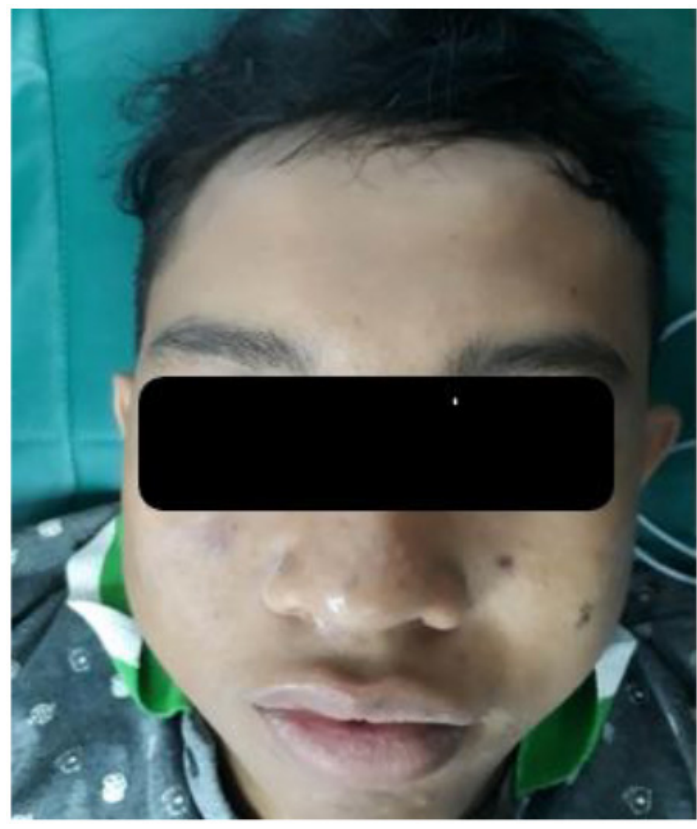

Figure 4. Clinical Photo

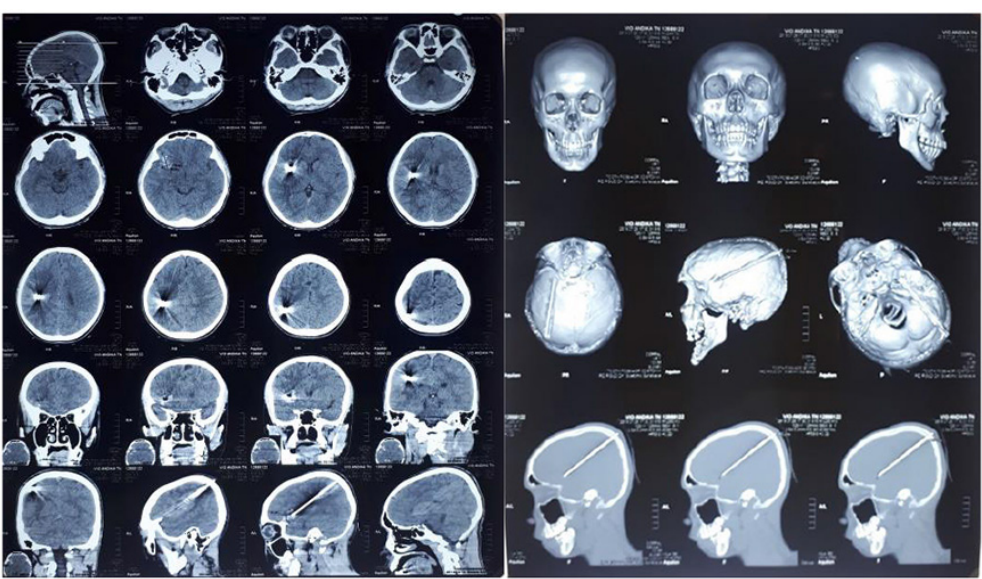

Figure 5. Head CT Scan

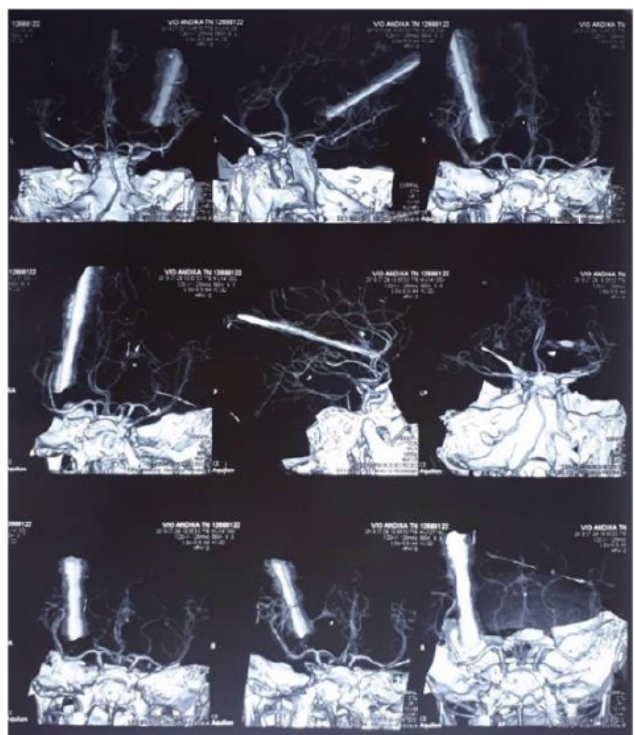

Figure 6. CT Angiography metronidazole 500mg / 8 hours with clinical evaluation of signs of infection. Phenytoin intravenously was given as seizure prophylaxis for 7 days. Patient was hospitalized for 9 days and there were no signs of intracranial infection and vision improved from light prophylaxis (+) to 6/10. The patient was then discharged with antibiotic cefixime $100 \mathrm{mg} / 12$ hours and metronidazole $500 \mathrm{mg} / 8$ hours per oral for 1 week.

\section{CASE 2}

Men, 18 years old, came to the emergency room with complaints of pain in the right eye since 2 hours before being admitted to the hospital. Pain was felt due to the right eye being shot by a fish bullet when the patient was reload a bullet. About $15 \mathrm{~cm}$ long fish bullet was suddenly shot at a distance of 20 $\mathrm{cm}$ in the patient's right eye. History of fainting (-), nausea (-), vomiting (-), fever (-), seizures (-).

Physical examination, primary survey and hemodynamically stable were obtained. On examination of the localis status, fish bullet was still stuck in the patient's right eye, there were no visible wounds. From neurological examination, GCS 456 was obtained, right eye anisocorous pupils were difficult to evaluate; left eye $3 \mathrm{~mm}$, light reflex right eye (-) left eye (-), visual acuity right eye was light perception (-) left eye $>2 / 60$, meningeal sign $(-)$, other neurological status within normal limits.

A head CT scan without contrast and CT angiography was performed. From head contrast without CT scan, the foreign body is found, the fish bullet penetrated the right orbital cavity and enter the right anterior fossa posteriorly until it enters the right occipital region accompanied by contour bleeding along the fish bullet path in the right temporal region. There were no bone fragments along the fish bullet path. From CT angiography no large blood vessels were involved and no traumatic aneurysm was present.

Patient was diagnosed with penetrating brain injury right temporoparietal region + right temporal contusion + extra ocular trauma. The foreign extraction and debridement + craniotomy were performed. The opthalmologist performed corneal repair and right ocular exploration..

The patient was positioned supine with his head turned to the left, blocking his right shoulder. Disinfection with povidone iodine and narrow the operating field with sterile linen. Pterional skin incision layer by layer until zygoma appears. Neurourgeon did burrhole 4 holes and craniotomy were performed. Preservation of the orbital supra nerves with chisel. One-piece orbitozygoma bone opening was performed, circumference of the circumference, bullet entry dura incision and intra 

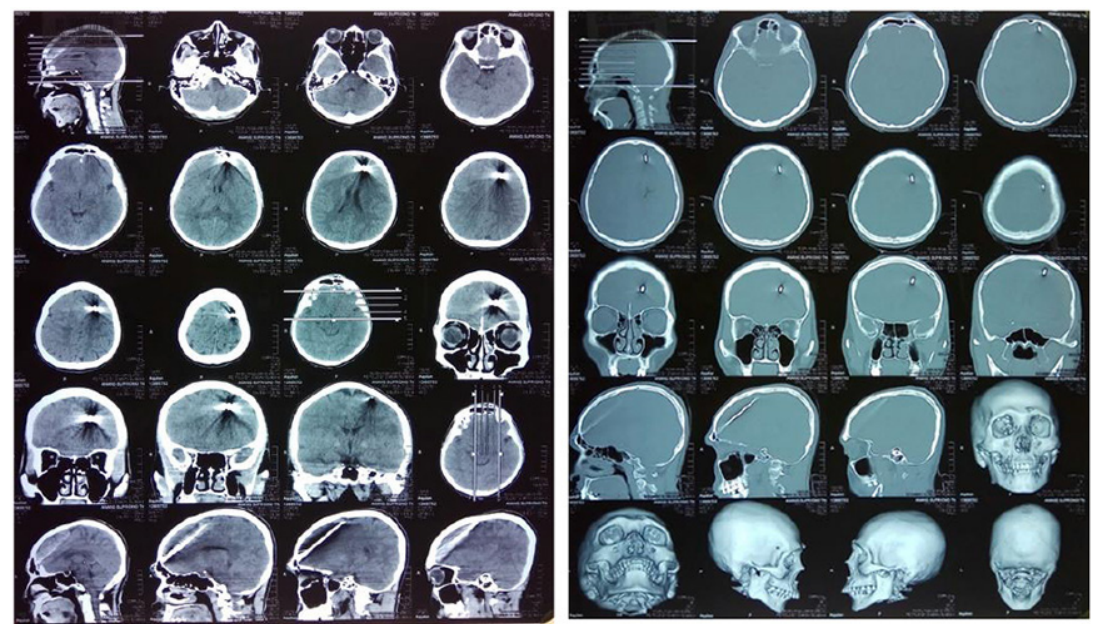

Figure 7. Head CT Scan bilateral isocorous pupils, light reflexes were postive in botheyes, meningeal sign (-), other neurological status within normal limit.

In patients then a head CT scan is performed without contrast. From a head scan without contrast, a picture of the foreign body could be found that the bullet pierces the left frontal sinus and pierces the frontal lobe.

Patients were diagnosed with penetrating brain left on frontoparietal region + open left posterior anterior sinus fracture. Foreign body extraction, craniotomy, debridement, decortication and decranialization were performed.

The patient was positioned supine, disinfected with povidone iodine and narrows the operating field with a sterile linen. Coronal incisions were carried out layer by layer of skin flap and perios dissection. Drilling bones around the foreign body until free, slowly extracting the corpus. After pulling out the bullets the 6 hole burrhole and craniotomy in the os frontal were carried out. Exploration of the dura and sewing of the defect in the dura mater were then carried out by decortication, decranization of the frontal sinus and osteoplasty. The wound was sutured layer by layer.

Culture samples were taken when debridement was carried out. Prophylactic empiric antibiotics were given to prevent infection with ceftriaxone inravena $1 \mathrm{~g} / 12$ hours and metronidazole 500mg / 8 hours with clinical evaluation of signs of infection. For seizure prophylaxis was given phenytoin for 7 days. Until treatment on the 7th day the patients did not get signs of intracranial infection. The patient was then discharged with oral antibiotics cefixime 100mg / 12 hours and metronidazole 500mg / 8 hours until the 14 th day postoperatively. clindamycin, levofloxacin.

Until the treatment of the 13th day the patients did not have signs of intracranial infection. The patient was then discharged with cefixime antibiotics $100 \mathrm{mg} / 12$ hours and metronidazole $500 \mathrm{mg} / 8$ hours per oral.

\section{CASE 3}

Man, 36 years old, came to the emergency room with a complaint that the patient's head had been shot by a fish bullet 12 hours before being hospitalized. A $15 \mathrm{~cm}$ long fish bullet was accidentally shot when the patient was pumping his gun. Decreased consciousness (-), nausea (-), vomiting (-), seizures $(-)$, fever $(+)$.

Physical examination, primary survey and hemodynamically stable were obtained. On examination of local status, fish bullet was stuck on the patient's forehead. From the neurological examination GCS 456 was obtained, $3 \mathrm{~mm} / 3 \mathrm{~mm}$

\section{DISCUSSION}

PBI is the most deadly type of head trauma. Every year in America around 32,000-35,000 residents die from PBI, with the most common cause being firearms. ${ }^{3}$

The degree of severity in penetrating brain trauma depends on how large the primary injury is. The ability of an object to be able to penetrate into brain tissue and cause primary brain injury depends on the nature of the ballistics, namely the kinetic energy produced, the mass of the object, speed, shape, angle of approach, the characteristics of objects that injure. ${ }^{7}$

If the speed of an object exceeds $700 \mathrm{~m} / \mathrm{s}$, the ability to injure will increase and can cause injury to the brain that is heavier and the increasing number of bone fragments that spread. ${ }^{7}$

In brain tissue that occurs penetration injury there is a temporary cavity and will form a shear- 
like injury to the neuron or even epidural, subdural or contusive bleeding in the brain parenchyma. ${ }^{7}$

The consequences of penetrating injury to the brain depend on the following factors: ${ }^{5}$

- Intracranial location (entry point): The high mortality rate is caused by penetrating lesions that pass through the midline, penetrate the brain ventricles, or to penetrate into the posterior fossa.

- Power and speed of objects: These penetration lesions can be formed depending on how much energy and speed are delivered to the skull and brain tissue below. The speed of an object can affect the mortality rate that occurs.

- Size

- Other accompanying injuries

Brain tissue has a high concentration of water so it has a relatively elastic shape and is prone to penetrating injuries. The high pressure from an object will cause crush injury and cell necrosis along the lesion that forms a cavity. The cause of the formation of this cavity is the high calm and speed of an object and cell death due to stretching, shearing, and compression of the surrounding brain tissue. ${ }^{4}$

Management of PBI starts from the primary survey and stabilizes airway, breathing, and circulation. Evaluation and resuscitation must be done immediately after the patient arrives at the emergency room. After the patient is resuscitated, evaluation of the head injury is immediately performed. Entry wounds must be identified immediately and the presence or absence of exit wounds must also be evaluated. Check for presence of cerebrospinal fluid, blood, or brain parenchyma that appears from the wound. Examination of neurological status is carried out after the evaluation of the wound is complete followed by a status general examination to look for any abnormalities in other organs. ${ }^{3}$

Laboratory tests include analysis of blood gases, electrolytes, complete blood, coagulation factors, blood type, alcohol and drugs. After the laboratory examination is completed the patient is immediately taken to radiology for further examination. ${ }^{3}$

Plain head photo can be useful in determining the location of foreign objects in the head, the presence or absence of bone fragments and intracranial air. However, plain photo inspection cannot be used to evaluate other important things such as bleeding and projectile trajectories. Therefore examination is recommended as the main radiological support because it can provide more complete information with a relatively short examination time. ${ }^{1}$ CT angiography can greatly help detect vascular lesions in the form of bleeding or traumatic aneurysms. ${ }^{4}$
In most cases, magnetic resonance imaging (MRI) examination is not recommended because of its limitations in detecting foreign objects, prolonged exposure time and contraindication if the projectile is made of metal. ${ }^{1,2,4}$ Surgical therapy in penetrating brain trauma has developed over time. Before 1889, in brain penetrating trauma patients there was no operative action because there was no homeostasis modality and bad infection prevention. Harvey Cushing was the first to develop an operating technique and to carry out bullet removal in a head trauma patient. In addition, Cushing also debrides and decompresses craniectomy. ${ }^{3}$

Surgery is best done in less than 12 hours. Debridement measures more than 12 hours increase the risk of infection. The choice of operative action with craniotomy or craniectomy is still debated. Radical debridement became a standard in World War I and II, Korea, and Vietnam. In 1980 during the Israel-Lebanon conflict the trend of debridement in penetrating head trauma shifted to conservative debridement with the aim of maintaining as much brain tissue as possible. ${ }^{3}$

Clinical factors associated with poor prognosis include low post-resuscitation GCS, old age, large and asymmetrical pupils and unreactive, hypoxic, and hypotensive (systolic pressure $<90 \mathrm{mmHg}$ ). GCS is generally considered the best single predictor for good or bad prognosis. ${ }^{8-10}$

Infection is a complication that is not uncommon in penetrating brain trauma patients, the presence of infection increases the mortality and morbidity of patients. The risk of infection is mainly due to contamination from foreign objects, skin, hair, and bone fragments that are pushed into brain tissue along the projectile pathway. ${ }^{2}$

Staphylococcus aureus is a type of organism that is often the cause of infection in penetrating brain trauma. But infection with gram negative bacteria is also quite common. Giving broad-spectrum antibiotics must be started as soon as possible in all penetrating brain trauma patients. The recommended choice of antibiotics is $1.2 \mathrm{~g} / 8 \mathrm{~h}$ coamoxiclav, or cefuroxime iv $1.5 \mathrm{~g}$ and maintenance $750 \mathrm{mg} /$ 8hours with metronidazole iv 500mg / 8hours given intravenously. Antibiotics are given for 7-14 days. ${ }^{2,3}$

There are many complications that may occur in PBI. One of the most frequent and severe cases is cerebral vasospasm due to subarachnoid haemorrhage and traumatic aneurysm. Cerebral vasospasm usually occurs between days 5-11 post trauma. Therefore CT angiography is recommended in cases where there is a suspicion of vascular lesions. ${ }^{1,3}$ Another factor is the strength of the weapon, caliber of weapons, lesions on important structures of CT scans. ${ }^{3}$ 
Of the 3 cases that all patients had GCS came 15 without encountering a neurological deficit, this was caused by penetrating brain trauma which occurred due to low velocity weapons and did not hit important areas of the brain. In all three cases a head CT scan was performed to determine the position of the foreign body in the brain and its relationship with important structures. CT angiography was also performed in case 1 and 2 to assess the presence or absence of vascular lesions and the possibility of traumatic aneurysm complications. In the third patient because the results of the initial head CT scan were estimated not to have an important vascular structure, CT angiography is not performed. CT angiographic of patients 1 and 2 revealed no vascular lesions.

Plain head photo examination was not peformed because the information obtained from the CT scan was sufficient. In all cases empirical and antiseizure antibiotics were carried out since the patient came to the emergency room. Craniotomy and debridement were performed in less than 12 hours onset of events according to existing guidelines.

The empirical antibiotics given were ceftriaxone and metronidazole as soon as the patient arrives and continues for 14 days. All patients were discharged in a stable condition and were asked to control every month for follow-up of the signs of infection for 6 months. All the three patients in the 3 months follow up showed no signs of infection.

\section{CONCLUSSION}

In our experience by following the trauma guidelines in Dr. Hospital Soetomo found that in PBI patients who performed debridement craniotomy less than 12 hours post trauma followed by the administration of empirical prophylactic antibiotics with ceftriaxone and metronidazole for 7 days and antiperspirants of phenytoin for 7 days obtained satisfactory outcome.

Head CT scan is mandatory for diagnosis of PBI, whereas plain photo examination does not need to be routine because its function can be replaced by CT scans. CT angiography is needed in cases of suspected vascular lesions.

\section{CONFLICT OF INTEREST}

There is no conflict of interest related to the materials or methods used in this study.

\section{FUNDING}

This article received no specific funding from any funding agency in the public, commercial, or notfor-profit sectors.

\section{AUTHORS' CONTRIBUTIONS}

Authors took part in design of the study, contributed to data collection, participated in writing the manuscript and all agree to accept equal responsibility for accuracy of the contents of this article.

\section{REFERENCES}

1. Temple N, Donald C, Skora A et al. Neuroimaging in Adult Penetrating Brain Injury: A Guide for Radiographers. Journal of Medical Radiation Science. 2015; 62:122-131

2. Kazim FS, Shamim SM, Tahir ZM et al. Management of Penetrating Brain Injury. Journal of Emergencies, Trauma, and Shock. 2011; 4(3): 395-402

3. Wyck VWD, Grant AG, Laskowitz TD. Penetrating Traumatic Brain Injury: A Review of Current Evaluation and Management Concepts. Journal of Neurology \& Neurophysiology. 2015; 6(6): 336

4. Vakil, M. T., \& Singh, A. K. A review of penetrating brain trauma: epidemiology, pathophysiology, imaging assessment, complications, and treatment. Emergency Radiology. 2017; 24(3), 301-309.

5. Alao T, Waseem M. Penetrating Head Trauma. StatPearls Publishing 2018.

6. Shi J, Mao Y, Cao J et al. Management of ScrewdriverInduced Penetrating Brain Injury: A Case Report. BMC Surgery. 2017; 17-3

7. Miranda ARH, Rubiano MA, Agrawal A et al. Craiocerebral Gunshot Injuries: A review of The Current Literature. Bulletin of Emergency and Trauma. 2016; 4(2): 65-74

8. Rosenfeld JV, Bell RS, Armonda R. Current concepts in penetrating and blast injury to the central nervous system. World J Surg. 2015; 39: 1352-1362.

9. Joseph B, Aziz H, Pandit V, Kulvatunyou N, O’Keeffe T, et al. Improving survival rates after civilian gunshot wounds to the brain. J Am Coll Surg. 2014; 218: 58-65.

10. Aarabi B, Tofighi B, Kufera JA, Hadley J, Ahn ES, et al. Predictors of outcome in civilian gunshot wounds to the head. J Neurosurg. 2014; 120: 1138-1146.

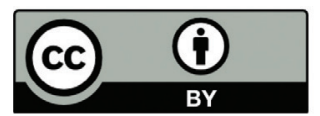

This work is licensed under a Creative Commons Attribution 